\title{
Correction to: Effects of miR-150-5p on the growth and SOCS1 expression of rheumatoid arthritis synovial fibroblasts
}

\author{
Mingliang Qiu ${ }^{1,2} \cdot{\text { Lisha } \mathrm{Mo}^{2} \cdot \text { Juxiang } \mathrm{Li}^{1} \cdot \text { Hua Liang }}^{3} \cdot$ Weina Zhu ${ }^{4} \cdot$ Xiangjuan Zheng $^{5} \cdot$ Xinwang Duan $^{6}$. \\ Weidong $\mathrm{Xu}^{2}$
}

Published online: 16 January 2020

(C) International League of Associations for Rheumatology (ILAR) 2020

\section{Correction to: Clinical Rheumatology https://doi.org/10.1007/s10067-019-04894-7}

The first name of the co-author of the above article was presented incorrect in the published version. The author name "Miangliang Qiu" should read "Mingliang Qiu" as mentioned above. The original article has been corrected.

The online version of the original article can be found at https://doi.org/ 10.1007/s10067-019-04894-7

Xinwang Duan

13970085678@163.com

Weidong $\mathrm{Xu}$

jindadong169@163.com

Mingliang Qiu

mingliangqiu@163.com

Lisha Mo

molisha125@126.com

Juxiang Li

ljx912@126.com

Hua Liang

wjf-queen-2008@163.com

Weina Zhu

weinazhu123@163.com

Xiangjuan Zheng

zhengxiangjuan@ncu.edu.cn
1 Department of Cardiovascular Medicine, The Second Affiliated Hospital of Nanchang University, Nanchang 330006, China

2 Department of Rheumatology, The Affiliated Hospital of Jiangxi University of Traditional Chinese Medicine, Nanchang 330006, China

3 Department of Clinical Laboratory, The Affiliated Hospital of Jiangxi University of Traditional Chinese Medicine, Nanchang 330006, China

4 Department of Pediatrics, The Affiliated Hospital of Jiangxi University of Traditional Chinese Medicine, Nanchang 330006, China

5 Department of Chemistry, Nanchang University, Nanchang 330031, China

6 Department of Rheumatology, The Second Affiliated Hospital of Nanchang University, Nanchang 330006, China 